\title{
Peripheral inflammatory markers in patients with prolonged disorder of consciousness after severe traumatic brain injury
}

\author{
Xiaohong Wu, Wen Xu, Tianfang Zhang, Wangxiao Bao \\ Department of Rehabilitation Medicine, First Affiliated Hospital, Zhejiang University School of Medicine, Hangzhou, China \\ Contributions: (I) Conception and design: X Wu, W Bao; (II) Administrative support: W Xu, T Zhang; (III) Provision of study materials or patients: \\ W Bao; (IV) Collection and assembly of data: T Zhang, W Bao; (V) Data analysis and interpretation: X Wu, T Zhang; (VI) Manuscript writing: All \\ authors; (VII) Final approval of manuscript: All authors. \\ Correspondence to: Wangxiao Bao. Department of Rehabilitation Medicine, First Affiliated Hospital, Zhejiang University School of Medicine, \#79 \\ Qingchun Road, Hangzhou, China. Email: baowangxiao@zju.edu.cn.
}

\begin{abstract}
Backgroundk Inflammatory processes are known to be the key secondary effects of severe traumatic brain injury (sTBI). The aim of the present study was to assess the value of peripheral inflammatory markers in the chronic unconscious phase after sTBI.

Methods: This was a prospective cohort study. A total of 101 patients with prolonged disorder of consciousness (DoC) and 22 healthy controls (HC) were enrolled in the study. Serum levels of interleukin (IL) $-1 \beta,-4,-6,-10,-13$, and tumor necrosis factor- $\alpha$ (TNF- $\alpha$ ) were investigated in patients with prolonged DoC after sTBI. In addition, the Coma Recovery Scale-revised (CRS-R) was used to quantify the consciousness level, and clinical outcomes at 12 months were determined using the Glasgow Outcome Scale (GOS). Predictive logistic model was built based on the demographic characteristics and cytokine levels.

Results: At baseline, IL-6, -10, -13 , and TNF- $\alpha$ levels were significantly higher in patients with prolonged DoC compared with controls, while no differences in cytokine levels were observed between patients in a vegetative state (VS) and those in a minimally conscious state (MCS). IL-13 and TNF- $\alpha$ were found to be correlated with behavioral scores in patients with prolonged DoC, and were associated with recovery 12 months later.

Conclusions: The results of the study provide information about long-term inflammatory responses in the chronic unconscious phase after brain trauma. Further larger studies are required to validate the value of these inflammatory markers.
\end{abstract}

Keywords: Severe traumatic brain injury (sTBI); prolonged disorder of consciousness (prolonged DoC); inflammation; recovery

Submitted Jun 28, 2021. Accepted for publication Jul 26, 2021.

doi: 10.21037/apm-21-1852

View this article at: https://dx.doi.org/10.21037/apm-21-1852

\section{Introduction}

Prolonged disorder of consciousness (DoC), also known as chronic DoC, is most frequently caused by severe traumatic brain injury (sTBI) and remained unconscious for more than 1 month, mainly includes a vegetative state (VS)/ unresponsive wakefulness syndrome (UWS) and a minimally conscious state (MCS) $(1,2)$. Previous studies have reported that patients in a traumatic VS for more than 1 year might remain in an unconscious state permanently (3). As for now, the Coma Recovery Scale-revised (CRS-R) is still considered to be the best behavioral scale for differential diagnosis of DoC, even though the misdiagnosis rate has reached $30-40 \%$. In the last few years, new techniques have been developed to improve the diagnostic and prognostic value. Behavioral assessments, multimodal neuroimaging methods, and fluid biomarkers (cerebrospinal fluid, serum and plasma) have been reported as the potential predictors 
of recovery in DoC patients.

In addition to the primary local impact injury that occurs immediately upon trauma, secondary brain injury becomes progressively exacerbated during the post-injury period (4). Recently, there has been significant insight into the pathobiology of secondary injury, revealing that such injury involves metabolic disturbances, excitotoxicity, oxidative stress, inflammation, and apoptosis $(5,6)$. Previous studies from our laboratory have shown that the inflammatory response plays a vital role in the chronic unconscious phase after sTBI (7). However, evidence of a relationship between the inflammatory response and DoC has been inconclusive.

Investigators have recently reported the dual roles of central and peripheral inflammation in TBI $(8,9)$. Cytokines, a group of inflammatory mediators, have multiple functions and can induce, exacerbate, mediate, and inhibit subsequent pathophysiological processes in the injured brain $(10,11)$. More specifically, high levels of inflammatory cytokines, such as interleukin (IL) $-1 \beta,-4,-6,-10$, and -13 , as well as tumor necrosis factor- $\alpha$ (TNF- $\alpha$ ) have been reported in the peripheral blood stream after sTBI (12-14). IL-1 $\beta$, -6 , and TNF- $\alpha$ are pro-inflammatory cytokines, whereas IL-4, -10, and -13 perform anti-inflammatory functions. A number of researchers have evaluated the effects of inflammatory cytokines during the acute phase $(15,16)$, the inflammatory cytokines not only induce secondary injury and neurodegeneration but also promote neural repair and functional recovery, however, uncertainty still exists about the roles of inflammatory cytokines during the chronic phase after sTBI.

The present study was designed to examine whether serum inflammatory markers are associated with clinical performance and long-term outcomes in patients with $\mathrm{DoC}$ after sTBI. The levels of inflammatory cytokines IL-1 $\beta,-4$, -6 , IL-10, -13, and TNF- $\alpha$ were compared between patients with VS/UWS and patients with MCS. We hypothesized that peripheral inflammatory markers offer clinically valuable information on the chronic unconscious phase after sTBI. We present the following article in accordance with the MDAR reporting checklist (available at https://dx.doi. org/10.21037/apm-21-1852).

\section{Methods}

\section{Patients}

This prospective cohort study enrolled 101 patients with prolonged DoC admitted to the rehabilitation units of Hangzhou Wujing Hospital (Hangzhou, Zhejiang, China) from January 2015 to January 2018. All patients had suffered severe brain trauma and remained unconscious for more than 1 month. Patients with acute infectious diseases or liver dysfunction were excluded. The location of the region showing structural damage and any clinical complications following the trauma were carefully recorded. Twenty-two age- and sex-matched individuals participated in the study as healthy controls (HC).

All procedures performed in this study involving human participants were in accordance with the Declaration of Helsinki (as revised in 2013). The study was approved by the Ethical Committee of the First Affiliated Hospital, Zhejiang University School of Medicine (No. 2015_310). Written informed consent was obtained from the patients' legal guardians. The trial was registered on ClinicalTrials. gov (ID No. NCT03908541).

\section{Blood collection}

Venous blood samples $(5 \mathrm{~mL})$ were collected from each patient using standardized phlebotomy procedures. Blood samples were collected in a Vacutainer tube without anticoagulant, centrifuged at $2,000 \times \mathrm{g}$ for $5 \mathrm{~min}$ at $4^{\circ} \mathrm{C}$, and stored at $-80^{\circ} \mathrm{C}$ until further analysis.

\section{Measurement of inflammatory markers}

The serum concentrations of IL- $1 \beta,-4,-6,-10,-13$, and TNF- $\alpha$ were assessed using the MILLIPLEX MAP Kit (Millipore, Billerica, MA, USA). The data are expressed as picograms per milliliter. Cytokine sample processing and data analysis were performed according to the manufacturer's instructions. All measurements were performed at the same time on the same machine by one board-certified laboratory technician who was blinded to the patients' clinical characteristics.

\section{Behavioral assessment}

The CRS-R was used to separate the patients into the VS/UWS and MCS groups (17). The CRS-R consists of 23 hierarchically arranged items that constitute 6 subscales addressing auditory, visual, motor, oromotor/ verbal, communication, and arousal processes. The clinical outcome was determined using the Glasgow Outcome Scale (GOS) at 12 months' follow up and was 
Table 1 Baseline characteristic of the included individuals

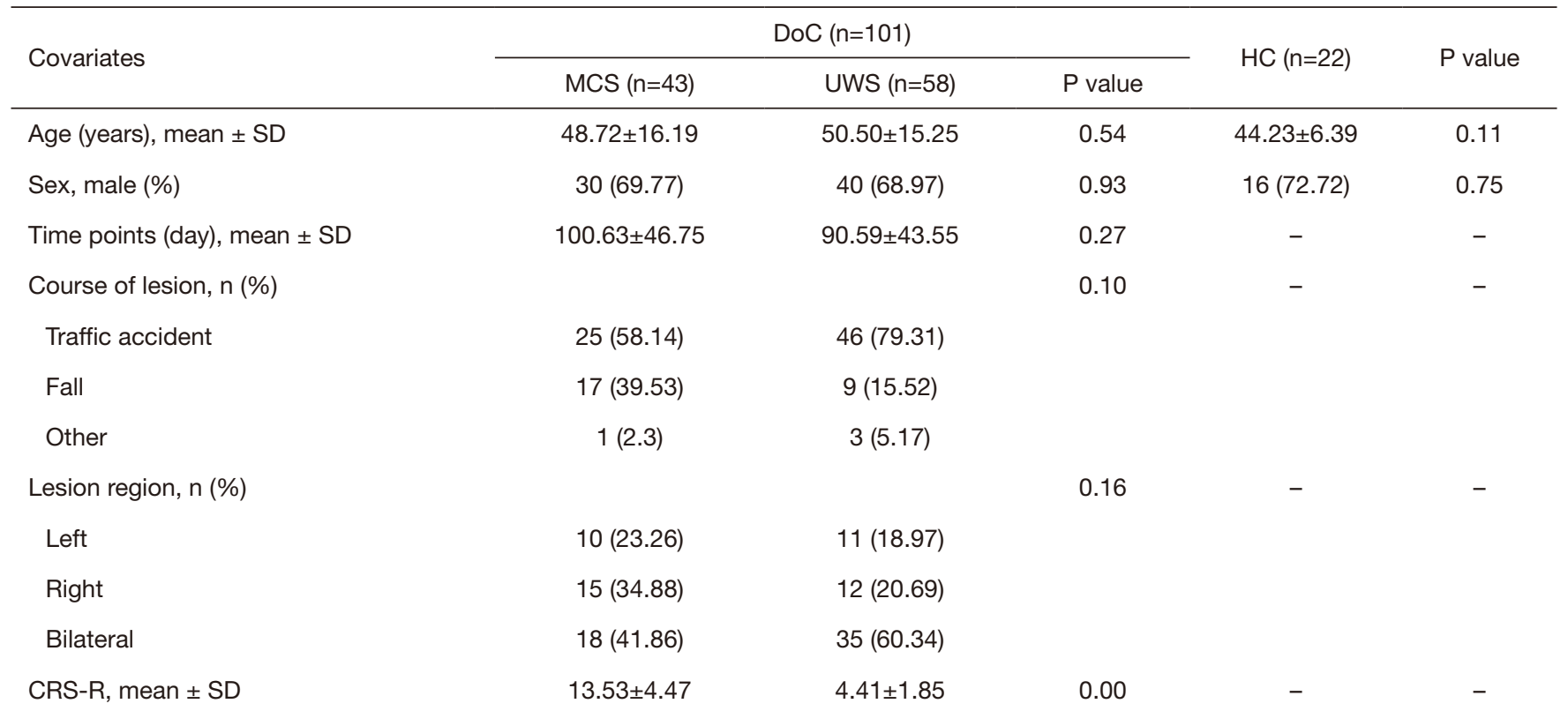

DoC, disorder of consciousness; MCS, minimally conscious state; UWS, unresponsive wakefulness syndrome; HC, healthy controls; SD, standard deviation; CRS-R, Coma Recovery Scale-revised.

dichotomized as favorable (GOS 3-5) or unfavorable (GOS $1-2$ ), where GOS $1=$ death, GOS 2= VS, GOS $3=$ severe disability, GOS 4= mild-to-moderate disability, and GOS $5=\operatorname{good}$ recovery $(18)$.

\section{Statistical analysis}

One-way analysis of variance was applied to compare cytokine levels between the patient and control groups. Correlations between cytokine levels and CRS-R scores were evaluated using the Spearman correlation coefficient. A binary logistic regression analysis was performed to determine associations between the variables and clinical recovery at 12 months. All $\mathrm{P}$ values presented are twotailed, and $\mathrm{P}<0.05$ was considered statistically significant.

\section{Results}

Of the 300 patients with DoC admitted to Wujing Hospital between January 2015 and January 2018, 101 met the inclusion criteria and were included in the present. Table 1 shows the demographic and clinical characteristics of the 101 patients and 22 controls. Patients with DoC had a mean age of 50 years and were predominantly male (69.3\%). At the baseline assessment, 43 DoC cases were classified as MCS (30 males, $48.72 \pm 16.19$ years) and 58 as

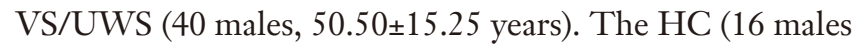
and 6 females) had a mean age of 44 years. There were no significant differences in age, sex, disease duration, disease cause, or lesion region among the MCS and VS/UWS groups.

The serum levels of peripheral inflammatory markers in the MCS, VS/UWS, and control groups are presented in Figure 1. Compared with controls, patients with MCS had significantly higher levels of IL-10 $(10.68 \pm 6.79 \mathrm{vs}$. $2.61 \pm 1.38 \mathrm{pg} / \mathrm{mL}, \mathrm{P}<0.001), \mathrm{IL}-13(3.42 \pm 2.48$ vs. $1.41 \pm$ $0.53 \mathrm{pg} / \mathrm{mL}, \mathrm{P}<0.001), \mathrm{IL}-1 \beta(1.19 \pm 0.64$ vs. $0.90 \pm$ $0.21 \mathrm{pg} / \mathrm{mL}, \mathrm{P}=0.04)$, IL-6 (4.29 \pm 3.72 vs. $0.97 \pm 0.68 \mathrm{pg} / \mathrm{mL}$, $\mathrm{P}=0.001)$, and TNF- $\alpha(33.25 \pm 11.77$ vs. $18.00 \pm 6.49 \mathrm{pg} / \mathrm{mL}$, $\mathrm{P}<0.001)$. Patients with VS/UWS had significantly higher levels of IL-10 $(8.91 \pm 5.20$ vs. $2.61 \pm 1.38 \mathrm{pg} / \mathrm{mL}, \mathrm{P}<0.001)$, IL-13 (2.64 \pm 2.35 vs. $1.41 \pm 0.53 \mathrm{pg} / \mathrm{mL}, \mathrm{P}=0.04)$, IL-6 $(4.61 \pm 3.90$ vs. $0.97 \pm 0.68 \mathrm{pg} / \mathrm{mL}, \mathrm{P}<0.001)$, and TNF- $\alpha$ (30.96 \pm 14.42 vs. $18.00 \pm 6.49 \mathrm{pg} / \mathrm{mL}, \mathrm{P}<0.001)$ compared with the controls. However, there were no significant differences in the inflammatory markers between MCS and VS/UWS patients.

The correlation coefficients between CRS-R scores and cytokine levels are shown in Figure 2. IL-13 and TNF- $\alpha$ concentrations were significantly correlated with CRS-R 

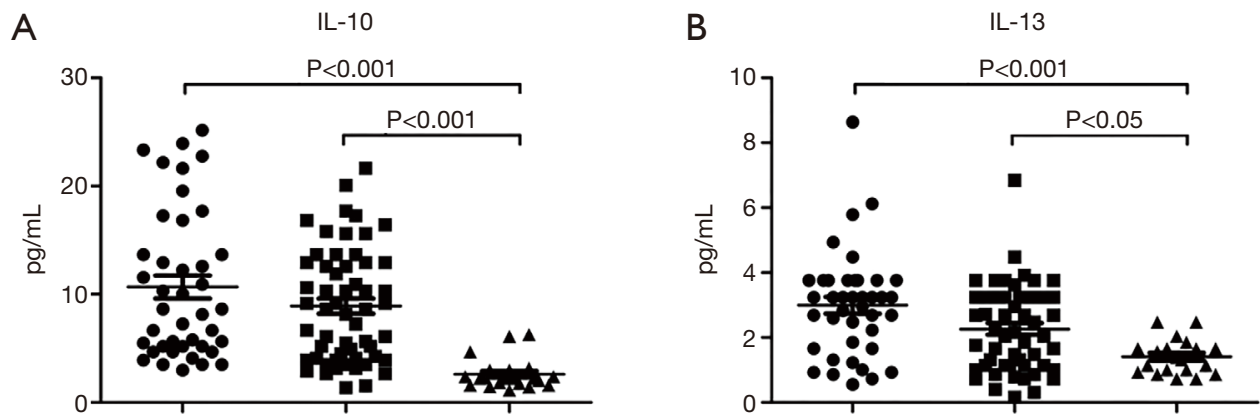

C

IL-1 $\beta$

D

IL-4
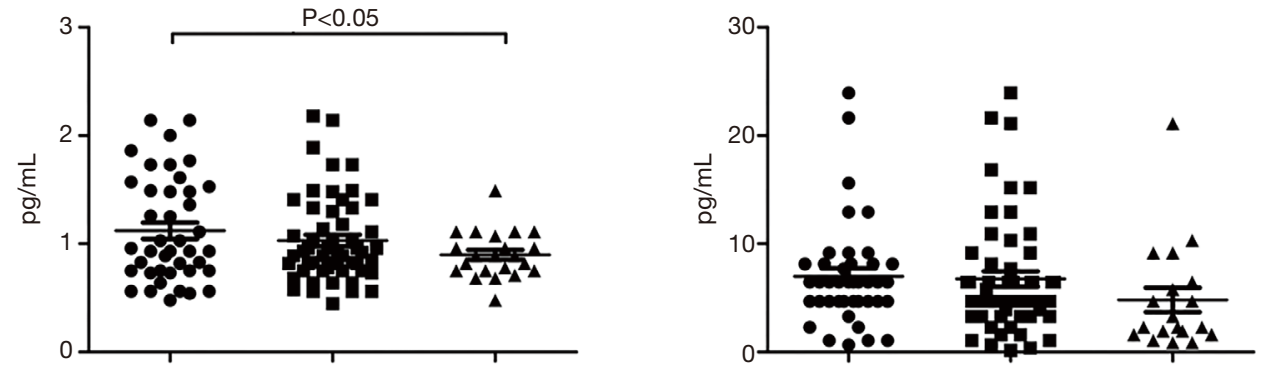

$E$

IL-6

F

TNF- $\alpha$
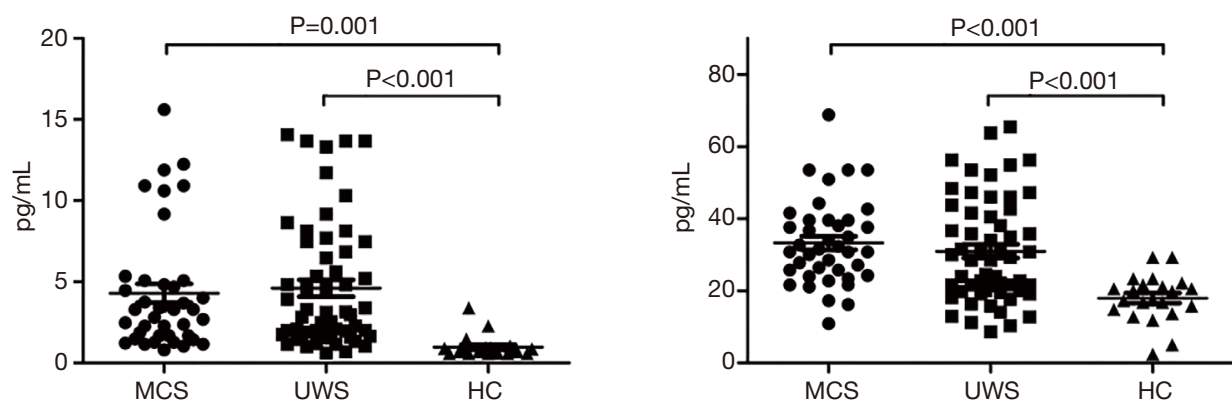

Figure 1 Serum inflammatory markers in patients with DoC after sTBI. Serum levels of (A) IL-10, (B) IL-13, (C) IL-1ß, (D) IL-4, (E) IL6 , and (F) TNF- $\alpha$ in the MCS, VS/UWS, and control groups. Statistical analysis was performed using one-way analysis of variance. Data are expressed as means $(\mathrm{pg} / \mathrm{mL})$. DoC, disorder of consciousness; sTBI, severe traumatic brain injury; IL, interleukin; TNF- $\alpha$, tumor necrosis factor- $\alpha$; MCS, minimally conscious state; VS, vegetative state; UWS, unresponsive wakefulness syndrome.

score, with correlation coefficients of $0.27(\mathrm{P}=0.002)$ and $0.23(\mathrm{P}=0.023)$, respectively (Figure 3). However, no other cytokine level was correlated with CRS-R score. In addition, IL-10 level was significantly correlated with levels of IL-6 and TNF- $\alpha$; IL-13 level was significantly correlated with levels of IL-4, -6 , and TNF- $\alpha$; IL-1 $\beta$ level was significantly correlated with levels of IL-4 and -6; and IL-6 level was significantly correlated with TNF- $\alpha$ level.

To examine the associations between the peripheral inflammatory markers and clinical recovery, logistic regression analysis was performed (Table 2). In this analysis, significant associations were found between IL-13 level and outcome [odds ratio (OR): 1.802, 95\% confidence interval (CI): 1.099-2.954, $\mathrm{P}=0.02]$ and TNF- $\alpha$ level and outcome (OR: 0.952, 95\% CI: 0.909-0.998, P=0.04). No statistically significant associations were found between age, sex, or time points.

\section{Discussion}

General pathophysiological cascades of secondary brain damage are initiated a few hours or even weeks after sTBI, 


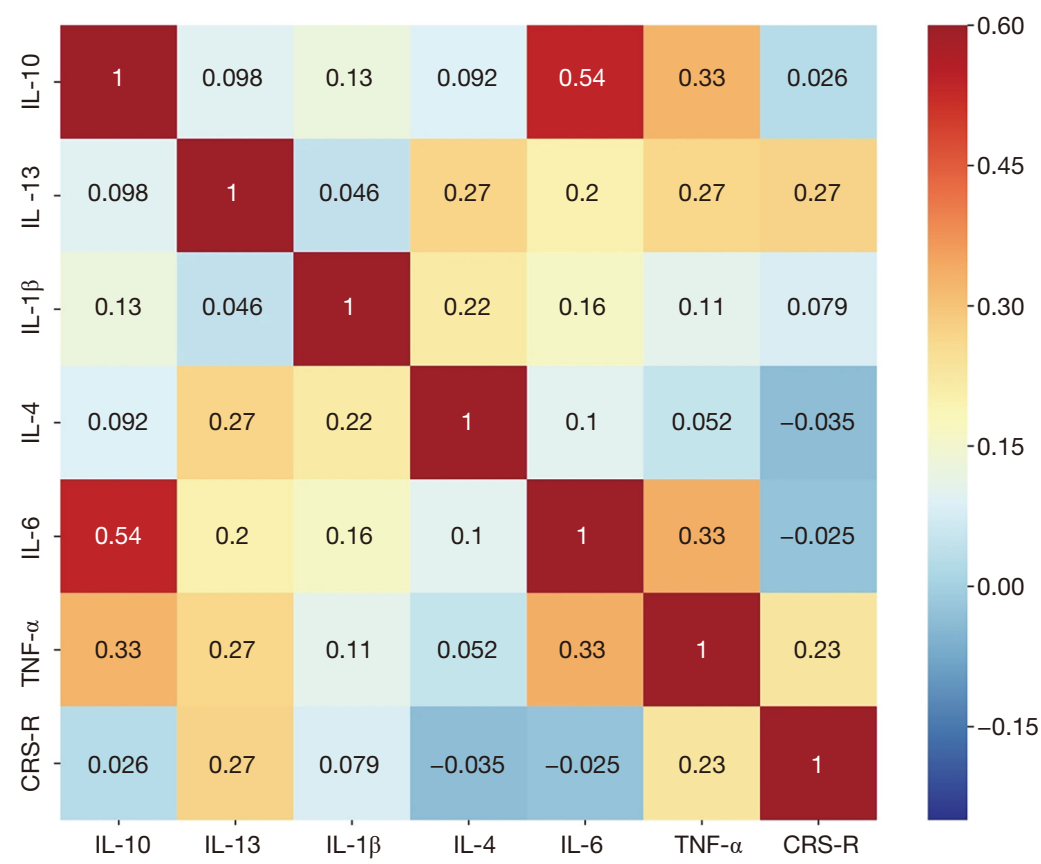

Figure 2 Correlation between the CRS-R score and cytokine levels. Correlations of serum levels of IL-1 $\beta,-4,-6,-10,-13$, and TNF- $\alpha$ with the CRS-R score were examined. CRS-R, Coma Recovery Scale-revised; IL, interleukin; TNF- $\alpha$, tumor necrosis factor- $\alpha$.
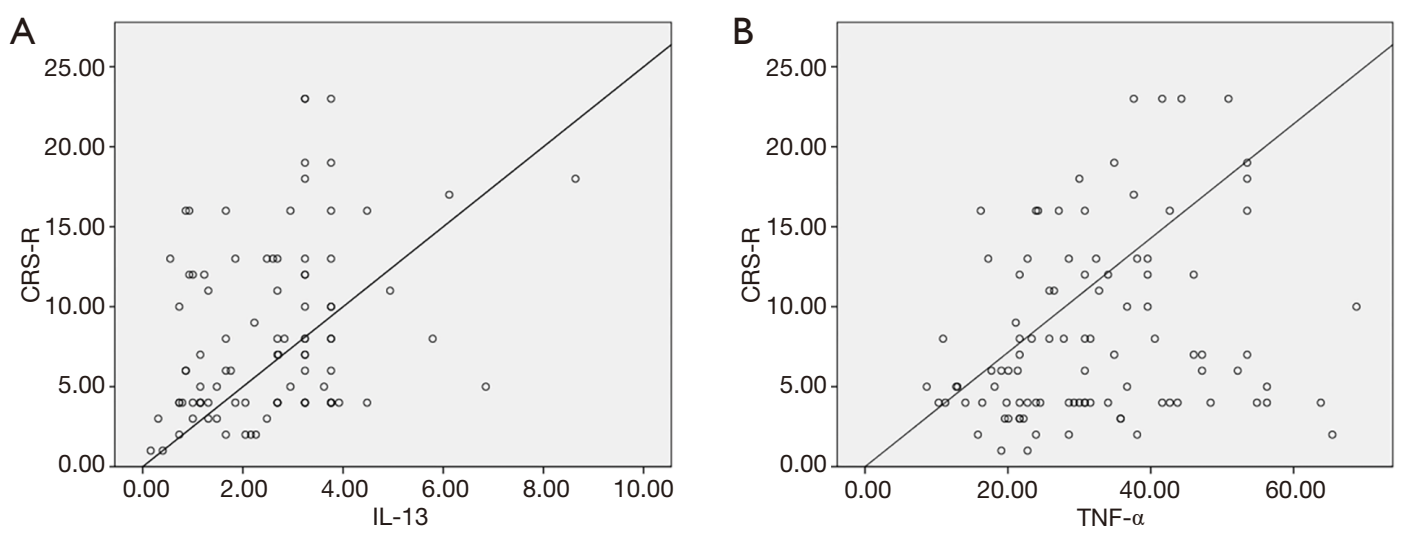

Figure 3 Association of (A) IL-13 and (B) TNF- $\alpha$ levels with the CRS-R score. IL, interleukin; TNF- $\alpha$, tumor necrosis factor- $\alpha$; CRS-R, Coma Recovery Scale-revised.

and an inflammatory response is a prominent component of these processes $(19,20)$. Inflammatory cytokines play central roles in the acute phase after sTBI (21); however, few studies have investigated long-term cytokine levels. Although TBI-related complications persist for years, little is known about the role of the inflammatory response in the chronic phase (22). To the best of our knowledge, the present study is the first to determine the effects of specific inflammatory markers in the chronic unconscious phase after sTBI.

A growing body of evidence suggests that cytokines play multiple roles in sTBI, depending on the time course and concentration $(10,23)$. In the present study, IL-10, -13, -6 , and TNF- $\alpha$ levels were significantly higher in patients with sTBI during the chronic unconscious phase than in controls, suggesting a persistent inflammatory response. 
Table 2 Binomial logistic regression analysis for the clinical recovery of patients with DoC according to demographic characteristics and cytokine levels

\begin{tabular}{lccc}
\hline Covariates & OR & $95 \% \mathrm{Cl}$ & $\mathrm{P}$ value \\
\hline Age & 0.994 & $0.955-1.034$ & 0.748 \\
Sex & 1.357 & $0.425-4.329$ & 0.606 \\
Time points & 0.993 & $0.979-1.007$ & 0.346 \\
IL-10 & 1.038 & $0.935-1.152$ & 0.489 \\
IL-13 & 1.802 & $1.099-2.954$ & $0.020^{*}$ \\
IL-1 $\beta$ & 1.764 & $0.476-6.530$ & 0.396 \\
IL-4 & 0.948 & $0.849-1.058$ & 0.340 \\
IL-6 & 0.889 & $0.742-1.067$ & 0.206 \\
TNF- $\alpha$ & 0.952 & $0.909-0.998$ & $0.041^{*}$ \\
\hline
\end{tabular}

*, statistical significance at $\mathrm{P}<0.05$. DoC, disorder of consciousness; OR, odds ratio; $\mathrm{Cl}$, confidence interval; IL, interleukin; TNF- $\alpha$, tumor necrosis factor- $\alpha$.

However, no significant differences in these cytokine levels were found between the VS/UWS and MCS groups, suggesting a lack of specificity in differentiating the degree of consciousness.

All of these cytokines have been reported to contribute to the inflammatory response following sTBI (24). IL-6, which performs pro-inflammatory functions, is a key effector in the damaged brain. Similarly, the plasma level of the antiinflammatory cytokine IL-10 was significantly higher in TBI patients and was also associated with the TBI outcome. Interestingly, an elevated IL-6/IL-10 ratio was previously found to be associated with outcome in TBI patients (9). The level of IL-4, a cytokine with both pro- and antiinflammatory properties, has been reported to increase after trauma and has been found to be more prominent in patients with favorable recovery (25). However, in the present study, no significant difference in IL-4 level was found in the chronic stage after sTBI.

Many previously published studies have reported that IL$6,-1,-8,-10$, and TNF- $\alpha$ levels are associated with worse outcomes $(11,24)$. In contrast, IL-13, a protective and antiinflammatory cytokine, has not been reported to be elevated in TBI studies. Although extensive studies have been carried out on inflammation after sTBI, the experimental data are unsatisfactory and controversial due to practical constraints. Inconsistent with the previous studies, our results indicated that specific inflammatory cytokines may be predictors of unfavorable outcomes in patients with DoC after sTBI.
Prolonged inflammation has been implicated in many chronic neurodegenerative diseases (26), whereas chronic inflammatory responses have not been well characterized following sTBI, especially in the chronic unconscious stage. It is important to understand the role of cytokines in the chronic phase after sTBI to improve the management of patients with DoC. Understanding the mechanisms underlying the disease course may offer novel intervention options, as well as therapeutic targets, for sTBI patients (27). One limitation of the present study was the small and homogeneous sample. Further studies involving a larger cohort of patients are needed to confirm our findings.

\section{Conclusions}

The findings of the present study indicated a peripheral inflammatory response during the late period after sTBI. Although chronic inflammatory activity has not been well characterized, several peripheral inflammatory markers could be used in long-term pathophysiological processes for sTBI. Future larger studies are required to further elucidate the value of these inflammatory markers.

\section{Acknowledgments}

Funding: This work was supported by the General Project of Health Department of Zhejiang Province (No. 2020370189).

\section{Footnote}

Reporting Checklist: The authors have completed the MDAR reporting checklist. Available at https://dx.doi. org/10.21037/apm-21-1852

Data Sharing Statement: Available at https://dx.doi. org/10.21037/apm-21-1852

Conflicts of Interest: All authors have completed the ICMJE uniform disclosure form (available at https://dx.doi. org/10.21037/apm-21-1852). The authors have no conflicts of interest to declare.

Ethical Statement: The authors are accountable for all aspects of the work in ensuring that questions related to the accuracy or integrity of any part of the work are appropriately investigated and resolved. All procedures performed in this study involving human participants were 
in accordance with the Declaration of Helsinki (as revised in 2013). The study was approved by the Ethical Committee of the First Affiliated Hospital, Zhejiang University School of Medicine (No. 2015_310). Written informed consent was obtained from the patients' legal guardians.

Open Access Statement: This is an Open Access article distributed in accordance with the Creative Commons Attribution-NonCommercial-NoDerivs 4.0 International License (CC BY-NC-ND 4.0), which permits the noncommercial replication and distribution of the article with the strict proviso that no changes or edits are made and the original work is properly cited (including links to both the formal publication through the relevant DOI and the license). See: https://creativecommons.org/licenses/by-nc-nd/4.0/.

\section{References}

1. Bernat JL. Chronic disorders of consciousness. Lancet 2006;367:1181-92.

2. Giacino JT, Katz DI, Schiff ND, et al. Practice guideline update recommendations summary: Disorders of consciousness: Report of the Guideline Development, Dissemination, and Implementation Subcommittee of the American Academy of Neurology; the American Congress of Rehabilitation Medicine; and the National Institute on Disability, Independent Living, and Rehabilitation Research. Neurology 2018;91:450-60.

3. Monti MM, Laureys S, Owen AM. The vegetative state. BMJ 2010;341:c3765.

4. Sahuquillo J, Poca MA, Amoros S. Current aspects of pathophysiology and cell dysfunction after severe head injury. Curr Pharm Des 2001;7:1475-503.

5. Ramlackhansingh AF, Brooks DJ, Greenwood RJ, et al. Inflammation after trauma: microglial activation and traumatic brain injury. Ann Neurol 2011;70:374-83.

6. Corps KN, Roth TL, McGavern DB. Inflammation and neuroprotection in traumatic brain injury. JAMA Neurol 2015;72:355-62.

7. Bao W, He F, Yu L, et al. Complement cascade on severe traumatic brain injury patients at the chronic unconscious stage: implication for pathogenesis. Expert Rev Mol Diagn 2018;18:761-6.

8. Balu R. Inflammation and immune system activation after traumatic brain injury. Curr Neurol Neurosci Rep 2014;14:484.

9. Kumar RG, Boles JA, Wagner AK. Chronic inflammation after severe traumatic brain injury: characterization and associations with outcome at 6 and 12 months postinjury. J Head Trauma Rehabil 2015;30:369-81.

10. Kadhim HJ, Duchateau J, Sébire G. Cytokines and brain injury: invited review. J Intensive Care Med 2008;23:236-49.

11. Rodney T, Osier N, Gill J. Pro- and anti-inflammatory biomarkers and traumatic brain injury outcomes: a review. Cytokine 2018;110:248-56.

12. Deepika A, Devi BI, Shukla D, et al. Neuroimmunology of traumatic brain injury: a longitudinal study of interdependency of inflammatory markers and heart rate variability in severe traumatic brain injury. J Neurotrauma 2018;35:1124-31.

13. Hensler T, Sauerland S, Riess P, et al. The effect of additional brain injury on systemic interleukin (IL)10 and IL-13 levels in trauma patients. Inflamm Res 2000;49:524-8.

14. Juengst SB, Kumar RG, Failla MD, et al. Acute inflammatory biomarker profiles predict depression risk following moderate to severe traumatic brain injury. J Head Trauma Rehabil 2015;30:207-18.

15. Arand M, Melzner H, Kinzl L, et al. Early inflammatory mediator response following isolated traumatic brain injury and other major trauma in humans. Langenbecks Arch Surg 2001;386:241-8.

16. Jastrow KM 3rd, Gonzalez EA, McGuire MF, et al. Early cytokine production risk stratifies trauma patients for multiple organ failure. J Am Coll Surg 2009;209:320-31.

17. Giacino JT, Kalmar K, Whyte J. The JFK Coma Recovery Scale-Revised: measurement characteristics and diagnostic utility. Arch Phys Med Rehabil 2004;85:2020-9.

18. Jennett B, Bond M. Assessment of outcome after severe brain damage. Lancet 1975;1:480-4.

19. Licastro F, Hrelia S, Porcellini E, et al. Peripheral inflammatory markers and antioxidant response during the post-acute and chronic phase after severe traumatic brain injury. Front Neurol 2016;7:189.

20. McDonald SJ, Sun M, Agoston DV, et al. The effect of concomitant peripheral injury on traumatic brain injury pathobiology and outcome. J Neuroinflammation 2016;13:90.

21. Savarraj J, Parsha K, Hergenroeder G, et al. Early brain injury associated with systemic inflammation after subarachnoid hemorrhage. Neurocrit Care 2018;28:203-11.

22. Maas AIR, Menon DK, Adelson PD, et al. Traumatic brain injury: integrated approaches to improve prevention, clinical care, and research. Lancet Neurol 
2017;16:987-1048.

23. Casault C, Al Sultan AS, Banoei M, et al. Cytokine responses in severe traumatic brain injury: where there is smoke, is there fire? Neurocrit Care 2019;30:22-32.

24. Di Battista AP, Rhind SG, Hutchison MG, et al. Inflammatory cytokine and chemokine profiles are associated with patient outcome and the hyperadrenergic state following acute brain injury. J Neuroinflammation 2016;13:40.

25. Majetschak M, Christensen B, Obertacke U, et al. Sex differences in posttraumatic cytokine release of

Cite this article as: $\mathrm{Wu} \mathrm{X}, \mathrm{Xu} \mathrm{W}$, Zhang T, Bao W. Peripheral inflammatory markers in patients with prolonged disorder of consciousness after severe traumatic brain injury. Ann Palliat Med 2021;10(8):9114-9121. doi: 10.21037/apm-21-1852 endotoxin-stimulated whole blood: relationship to the development of severe sepsis. J Trauma 2000;48:832-9; discussion 839-40.

26. Lucas SM, Rothwell NJ, Gibson RM. The role of inflammation in CNS injury and disease. Br J Pharmacol 2006;147 Suppl 1:S232-40.

27. Hazeldine J, Lord JM, Belli A. Traumatic brain injury and peripheral immune suppression: primer and prospectus. Front Neurol 2015;6:235.

(English Language Editor: R. Scott) 